\title{
QUANTUM ERGODICITY FOR A POINT SCATTERER ON THE THREE-DIMENSIONAL TORUS
}

\author{
NADAV YESHA
}

\begin{abstract}
Consider a point scatterer (the Laplacian perturbed by a delta-potential) on the standard three-dimensional flat torus. Together with the eigenfunctions of the Laplacian which vanish at the point, this operator has a set of new, perturbed eigenfunctions. In a recent paper, the author was able to show that all of the perturbed eigenfunctions are uniformly distributed in configuration space. In this paper we prove that almost all of these eigenfunctions are uniformly distributed in phase space, i.e. we prove quantum ergodicity for the subspace of the perturbed eigenfunctions. An analogue result for a point scatterer on the two-dimensional torus was recently proved by Kurlberg and Ueberschär.
\end{abstract}

\section{INTRODUCTION}

Consider a point scatterer on the standard three-dimensional flat torus $\mathbb{T}^{3}=\mathbb{R}^{3} / 2 \pi \mathbb{Z}^{3}$, which is formally given by

$$
-\Delta+\alpha \delta_{x_{0}}
$$

where $-\Delta$ is the associated Laplacian on $\mathbb{T}^{3}, \delta_{x_{0}}$ is the Dirac potential at $x_{0}$ and $\alpha$ is a coupling parameter.

We want to study quantum ergodicity of this system, which is a key question in the field of Quantum Chaos. A classical result regarding quantum ergodicity is Schnirelman's theorem [14, 4, 19, which asserts that for classically ergodic systems, the quantum counterpart is quantum ergodic, i.e. almost all eigenstates are uniformly distributed in phase space. However our system is not classically ergodic but an intermediate system; its classical dynamics is nearly integrable.

Rigorously, a point scatterer is obtained as a self-adjoint extension of the Laplacian acting on functions vanishing near $x_{0}$. Such extensions are parametrized by $\phi \in(-\pi, \pi]$, where $\phi=\pi$ corresponds to the standard Laplacian $(\alpha=0$ in (1.1) $)$. For $\phi \neq \pi$, the eigenfunctions of the corresponding operator consist of eigenfunctions of the Laplacian which vanish at $x_{0}$ (and are not affected by the scatterer, so they are related to the unperturbed problem), and new, perturbed eigenfunctions. Since the latter are the only eigenfunctions which feel the scatterer, we have to consider only them in order to study the physics of the perturbed problem.

In a recent paper [18, the author was able to prove quantum (unique) ergodicity regarding the perturbed eigenfunctions of a point scatterer on the 
standard three-dimensional flat torus, but only for observables which do not depend on the momentum. Our goal now is to prove quantum ergodicity regarding the perturbed eigenfunctions in full phase space.

We remark that a two-dimensional analogue of the theorem in [18] (i.e. quantum ergodicity in configuration space) was proved for a general twodimensional flat torus by Rudnick and Ueberschär [12]; in a recent work by Kurlberg and Ueberschär, they prove an analogue of the theorem in the current paper for the standard two-dimensional flat torus.

The unit (co)tangent bundle of $\mathbb{T}^{3}$ is the compact metric space $S^{*} \mathbb{T}^{3} \simeq$ $\mathbb{T}^{3} \times S^{2}$, on which we have the Liouville probability measure $\mu$ which is the normalized product of the Lebesgue measure $m$ on $\mathbb{T}^{3}$ and the Lebesgue measure $\sigma$ on $S^{2}$. Observables are smooth, zero-th order $\xi$-homogeneous functions $a(x, \xi)$ on $S^{*} \mathbb{T}^{3}$. In order to quantize them, we use the notion of pseudo-differential operators on $\mathbb{T}^{3}$, which will be discussed in greater detail in section 93 below. As we will see, quantizing $a \in C^{\infty}\left(S^{*} \mathbb{T}^{3}\right)$ leads to an operator $\operatorname{Op}(a)$, which is a bounded operator on $L^{2}\left(\mathbb{T}^{3}\right)$.

We now state the main theorem of this paper. For every $\phi \in(-\pi, \pi)$, let $\Lambda_{\phi}$ be the set of the perturbed eigenvalues of the point scatterer, with the corresponding $L^{2}$-normalized eigenfunctions $g_{\lambda}\left(\lambda \in \Lambda_{\phi}\right)$. We prove:

Theorem 1.1. Fix $\phi \in(-\pi, \pi)$. There is a subset $\Lambda_{\phi, \infty} \subseteq \Lambda_{\phi}$ of density one so that for all $a \in C^{\infty}\left(S^{*} \mathbb{T}^{3}\right)$,

$$
\left\langle\mathrm{Op}(a) g_{\lambda}, g_{\lambda}\right\rangle \rightarrow \int_{S^{*} \mathbb{T}^{3}} a \mathrm{~d} \mu
$$

as $\lambda \rightarrow \infty$ along $\Lambda_{\phi, \infty}$.

We actually prove a more general statement: let $\mathcal{N}_{3}$ be the set of integers which are sums of three squares (these are the eigenvalues of the Laplacian), and let $\Lambda$ be any increasing sequence whose elements interlace with the elements of $\mathcal{N}_{3}$. For any $\lambda \in \Lambda$, define $g_{\lambda}$ to be the $L^{2}$-normalized Green's function: $g_{\lambda}=\frac{G_{\lambda}}{\left\|G_{\lambda}\right\|_{2}}$, where $G_{\lambda}=(\Delta+\lambda)^{-1} \delta_{x_{0}}$.

Theorem 1.2. There is a subset $\Lambda_{\infty} \subseteq \Lambda$ of density one so that for all $a \in C^{\infty}\left(S^{*} \mathbb{T}^{3}\right)$,

$$
\left\langle\mathrm{Op}(a) g_{\lambda}, g_{\lambda}\right\rangle \rightarrow \int_{S^{*} \mathbb{T}^{3}} a \mathrm{~d} \mu
$$

as $\lambda \rightarrow \infty$ along $\Lambda_{\infty}$.

This is of interest since in the physics literature one considers $\phi$ which is not fixed, but varies as $\lambda \rightarrow \infty$ (see [15, 16]); since the only condition for $\Lambda$ in theorem 1.2 is that its elements interlace with the elements of $\mathcal{N}_{3}$, the theorem will still hold in such cases.

Approximating an observable with a linear combination of the functions $Y_{l, m}(\xi) e^{i x \cdot \zeta}$, where $Y_{l, m}$ are spherical harmonics, the question at hand reduces to an arithmetic one. The main case is $\zeta=0$ (the other cases will 
follow from the proof of proposition 3.9 in [18]), for which a new arithmetic ingredient is used - an estimate due to Duke, by which he proved in [5, 6] the equidistribution of integer lattice points on a sphere of radius $\sqrt{n}, n \not \equiv 0,4,7(8), n \rightarrow \infty$ (conjectured by Linnik, and proved independently by Golubeva and Fomenko [, 9]). It will be combined with Siegel's lower bound for $r_{3}(n)$, the number of representations of $n$ as a sum of three squares $(n \not \equiv 0,4,7(8))$ [17, which was also a key ingredient in proving the analogue result in configuration space in [18].

We comment that all this is very different from the two-dimensional case, where arithmetic questions about spheres are replaced by questions about circles. The two-dimensional analogue to Duke's estimate is the theorem of Erdős and Hall [7] about the distribution of lattice points on circles; an obvious complication constructing the density one sequence $\Lambda_{\infty}$ of perturbed eigenvalues is due to the fact that the theorem of Erdös and Hall is not deterministic, i.e. lattice points on circles are equidistributed only for an unspecified density one set of compatible $n$ 's.

Acknowledgments: This work is part of the author's Ph.D. thesis written under the supervision of Zeev Rudnick at Tel Aviv University. The author would like to thank him for suggesting the problem and for his useful ideas, discussions and comments. The author would also like to thank Stéphane Nonnenmacher for discussions concerning pseudo-differential operators.

The research leading to these results has received funding from the European Research Council under the European Union's Seventh Framework Programme (FP7/2007-2013) / ERC grant agreement nº 320755.

\section{Point Scatterers on the Torus}

Let $\mathbb{T}^{3}=\mathbb{R}^{3} / 2 \pi \mathbb{Z}^{3}$ be the standard flat torus. A rigorous definition of the operator (1.1) can be found in [18, following [3, 12]. For the convenience of the reader we give here a brief summary:

Let $D_{0}=C_{0}^{\infty}\left(\mathbb{T}^{3} \backslash\left\{x_{0}\right\}\right)$ be the domain of $C^{\infty}$ functions vanishing in a neighborhood of $x_{0}$, and define an operator on $L^{2}\left(\mathbb{T}^{3}\right)$ by $-\Delta_{x_{0}}=-\Delta_{\mid D_{0}}$. For the adjoint of $-\Delta_{x_{0}}$ we have

$$
\begin{aligned}
\operatorname{Dom}\left(-\Delta_{x_{0}}^{*}\right) & =H^{2}\left(\mathbb{T}^{3} \backslash\left\{x_{0}\right\}\right) \\
& =\left\{f \in L^{2}\left(\mathbb{T}^{3}\right): \exists A \in \mathbb{C},-\Delta f+A \delta_{x_{0}} \in L^{2}\left(\mathbb{T}^{3}\right)\right\},
\end{aligned}
$$

and the self-adjoint extensions of $-\Delta_{x_{0}}$ are indexed by a parameter $\phi \in$ $(-\pi, \pi]$; the domain of the corresponding operators $-\Delta_{\phi, x_{0}}$ contains the functions $f \in \operatorname{Dom}\left(-\Delta_{x_{0}}^{*}\right)$ such that

$$
\exists a \in \mathbb{C}, f(x)=a\left(\cos \frac{\phi}{2} \cdot \frac{-1}{4 \pi\left|x-x_{0}\right|}+\sin \frac{\phi}{2}\right)+o(1), \quad x \rightarrow x_{0}
$$

and their action on $f \in \operatorname{Dom}\left(-\Delta_{\phi, x_{0}}\right)$ is given by

$$
-\Delta_{\phi, x_{0}} f=-\Delta f+A \delta_{x_{0}}=-\Delta f+a \cos \frac{\phi}{2} \delta_{x_{0}}
$$


so we define a point scatterer to be one of these extended operators $-\Delta_{\phi, x_{0}}$.

We remark that for $\phi=\pi$ we have $\operatorname{Dom}\left(-\Delta_{\pi, x_{0}}\right)=H^{2}\left(\mathbb{T}^{3}\right)$ and $-\Delta_{\pi, x_{0}} f=$ $-\Delta f$, so this extension recovers the standard Laplacian $-\Delta_{\infty}$ on the domain $H^{2}\left(\mathbb{T}^{3}\right)$ (which is the unique self-adjoint extension of $-\Delta_{\mid C \infty\left(\mathbb{T}^{3}\right)}$ ).

The functions $(2 \pi)^{-3 / 2} e^{i \xi \cdot x}\left(\xi \in \mathbb{Z}^{3}\right)$ form an orthonormal basis of eigenfunctions of $-\Delta_{\infty}$ for $L^{2}\left(\mathbb{T}^{3}\right)$. The corresponding eigenvalues are the norms $|\xi|^{2}$ of vectors in $\mathbb{Z}^{3}$, i.e. the set $\mathcal{N}_{3}$ of integers which are sums of three squares, and each eigenvalue is of multiplicity $r_{3}(n)$ which is the number of representations of $n=a^{2}+b^{2}+c^{2}$ with $a, b, c \in \mathbb{Z}$ integers.

For the perturbed operator (2.1) with $\phi \neq \pi$ we still have the nonzero eigenvalues from the unperturbed problem $\left(0 \neq \lambda \in \sigma\left(-\Delta_{\infty}\right)\right)$, with multiplicities decreased by one, as well as a new set $\Lambda=\Lambda_{\phi}$ of eigenvalues (referred as the perturbed eigenvalues), each appearing with multiplicity one, with the corresponding eigenfunctions being multiples of the Green's function

$$
G_{\lambda}\left(x, x_{0}\right)=(\Delta+\lambda)^{-1} \delta_{x_{0}} .
$$

The main tool we use for studying the Green's functions $G_{\lambda}$ is their $L^{2}$ expansion, which is given by

$$
G_{\lambda}\left(x, x_{0}\right)=-\frac{1}{8 \pi^{3}} \sum_{\xi \in \mathbb{Z}^{3}} \frac{\exp \left(i \xi \cdot\left(x-x_{0}\right)\right)}{|\xi|^{2}-\lambda} .
$$

We denote by

$$
g_{\lambda}(x):=\frac{G_{\lambda}\left(x, x_{0}\right)}{\left\|G_{\lambda}\right\|_{2}}
$$

the $L^{2}$-normalized Green's function, or the normalized perturbed eigenfunctions of the scatterer.

One can see that perturbed eigenvalues are the solutions to the equation

$$
\sum_{\xi \in \mathbb{Z}^{3}}\left\{\frac{1}{|\xi|^{2}-\lambda}-\frac{|\xi|^{2}}{|\xi|^{4}+1}\right\}=c_{0} \tan \frac{\phi}{2}
$$

with

$$
c_{0}=\sum_{\xi \in \mathbb{Z}^{3}} \frac{1}{|\xi|^{4}+1}
$$

so the perturbed eigenvalues interlace with the elements of

$$
\mathcal{N}_{3}=\left\{0=n_{0}<n_{1}<n_{2}<\ldots\right\},
$$

and denoting them by $\lambda_{k}=\lambda_{k}^{\phi}$ we can write

$$
\lambda_{0}<n_{0}<\lambda_{1}<n_{1}<\lambda_{2}<\cdots<n_{k}<\lambda_{k+1}<n_{k+1}<\ldots
$$

Recall that a subset $\Lambda^{\prime}=\left\{\lambda_{j_{k}}\right\} \subseteq \Lambda$ is of density $a(0 \leq a \leq 1)$ in $\Lambda$ if

$$
\lim _{J \rightarrow \infty} \frac{1}{J} \#\left\{k \in \mathbb{N}: j_{k} \leq J\right\}=a
$$


or equivalently

$$
\lim _{X \rightarrow \infty} \frac{\#\left\{\lambda \in \Lambda^{\prime}: \lambda \leq X\right\}}{\#\{\lambda \in \Lambda: \lambda \leq X\}}=a
$$

\section{Pseudo-Differential Operators on the Torus}

In order to quantize observables $a \in C^{\infty}\left(S^{*} \mathbb{T}^{3}\right)$, one can use the special structure of $\mathbb{T}^{3}$ as a compact group, to get a global definition of pseudodifferential operators on $\mathbb{T}^{3}$, instead of using the theory of (Hörmander's) pseudo-differential operators on $\mathbb{R}^{3}$ locally, which could be rather inconvenient. This idea goes back to Agranovich [1], and it was proved by McLean [11] that both definitions of pseudo-differential operators on the torus are equivalent. A recent monograph by Ruzhansky and Turunen [13] gives a very comprehensive treatment of this subject - we present here the basic definitions of pseudo-differential operators on $\mathbb{T}^{n}$ (and in particular on $\mathbb{T}^{3}$ ) using their notations.

Let $\sigma: \mathbb{Z}^{n} \rightarrow \mathbb{C}$, and let $e_{j}$ be the standard basis elements of $\mathbb{R}^{n}$. Let $\Delta_{\xi_{j}}$ be the partial difference operator defined by

$$
\Delta_{\xi_{j}} \sigma(\xi)=\sigma\left(\xi+e_{j}\right)-\sigma(\xi),
$$

and for a multi-index $\alpha$ define

$$
\Delta_{\xi}^{\alpha}=\Delta_{\xi_{1}}^{\alpha_{1}} \cdots \Delta_{\xi_{n}}^{\alpha_{n}}
$$

Following the notation in [13], for $m \in \mathbb{R}, 0 \leq \delta, \rho \leq 1$, define $S_{\rho, \delta}^{m}\left(\mathbb{T}^{n} \times \mathbb{Z}^{n}\right)$ to be the set of functions $a(x, \xi)$ which are smooth in $x$ for all $\xi \in \mathbb{Z}^{n}$, and satisfy

$$
\left|\Delta_{\xi}^{\alpha} \partial_{x}^{\beta} a(x, \xi)\right| \leq C_{a, \alpha, \beta, m}\langle\xi\rangle^{m-\rho|\alpha|+\delta|\beta|}
$$

for every $x \in \mathbb{T}^{n}, \alpha, \beta$ multi-indices, and $\xi \in \mathbb{Z}^{n}$. Here $\langle\xi\rangle=\left(1+|\xi|^{2}\right)^{1 / 2}$.

For every symbol $a(x, \xi) \in S_{\rho, \delta}^{m}\left(\mathbb{T}^{n} \times \mathbb{R}^{n}\right)$, define a toroidal symbol $\widetilde{a}$ : $\mathbb{T}^{n} \times \mathbb{Z}^{n} \rightarrow \mathbb{C}$ by the restriction $\widetilde{a}=\left.a\right|_{\mathbb{T}^{n} \times \mathbb{Z}^{n}}$; it is not hard to show that $\widetilde{a}(x, \xi) \in S_{\rho, \delta}^{m}\left(\mathbb{T}^{n} \times \mathbb{Z}^{n}\right)$. Define

$$
\begin{aligned}
\operatorname{Op}(a) f(x) & =\operatorname{Op}(\widetilde{a}) f(x) \\
& =\sum_{\xi \in \mathbb{Z}^{n}} e^{i x \cdot \xi} a(x, \xi) \widehat{f}(\xi) .
\end{aligned}
$$

One verifies (see [13]) that the operator Op $(a): C^{\infty}\left(\mathbb{T}^{n}\right) \rightarrow C^{\infty}\left(\mathbb{T}^{n}\right)$ is well defined and continuous. Note that for a symbol $a(x, \xi)=\sum_{|\alpha| \leq m} a_{\alpha}(x) \xi^{\alpha}$ 
we get that

$$
\begin{aligned}
\text { Op }(a) f(x) & =\sum_{|\alpha| \leq m} a_{\alpha}(x) \sum_{\xi \in \mathbb{Z}^{3}} e^{i x \cdot \xi} \xi^{\alpha} \widehat{f}(\xi) \\
& =\sum_{|\alpha| \leq m} a_{\alpha}(x) \sum_{\xi \in \mathbb{Z}^{3}} e^{i x \cdot \xi} \widehat{(-i \partial)^{\alpha}} f(\xi) \\
& =\sum_{|\alpha| \leq m} a_{\alpha}(x)(-i \partial)^{\alpha} f(x)
\end{aligned}
$$

as one would expect.

In particular, we use this quantization for zero-th order positively homogeneous symbols $a(x, \xi) \in S_{1,0}^{0}\left(\mathbb{T}^{3} \times \mathbb{R}^{3}\right)$ (in the sense that $a(x, \lambda \xi)=a(x, \xi)$ for $|\xi| \geq 1$ and $\lambda \geq 1)$. Since $a(x, \xi)$ is a smooth $\xi$-homogeneous function of order zero, we can identify it with a smooth function on the unit cotangent bundle of $\mathbb{T}^{3}$, and we write $a \in C^{\infty}\left(S^{*} \mathbb{T}^{3}\right)$.

One proves that in this case, Op $(a)$ extends to a bounded operator on $L^{2}\left(\mathbb{T}^{3}\right)$. Moreover, we have the following result on the $L^{2}$-norm of Op $(a)$ (theorem 4.8.1 in [13]): Let $k \in \mathbb{N}$ and $k>n / 2$. Let $a: \mathbb{T}^{n} \times \mathbb{Z}^{n} \rightarrow \mathbb{C}$ be such that $\left|\partial_{x}^{\beta} a(x, \xi)\right| \leq C$ for all $(x, \xi) \in \mathbb{T}^{n} \times \mathbb{Z}^{n}$ and all $|\beta| \leq k$. Then the operator Op $(a)$ extends to a bounded linear operator on $L^{2}\left(\mathbb{T}^{n}\right)$, and there exists a constant $C_{k}$ (which depends only on $k$ ) such that

$$
\|\mathrm{Op}(a)\|_{L^{2}\left(\mathbb{T}^{n}\right) \rightarrow L^{2}\left(\mathbb{T}^{n}\right)}^{2} \leq C_{k} \sum_{|\alpha| \leq k} \sup _{y \in \mathbb{T}^{n}} \sup _{\xi \in \mathbb{Z}^{n}}\left|\partial_{y}^{\alpha} a(y, \xi)\right|^{2} .
$$

Thus, for $n=3, k=2, a \in S_{1,0}^{0}\left(\mathbb{T}^{3} \times \mathbb{R}^{3}\right)$ we have

$$
\|\mathrm{Op}(a)\|_{L^{2}\left(\mathbb{T}^{3}\right) \rightarrow L^{2}\left(\mathbb{T}^{3}\right)}^{2} \leq C \sum_{|\alpha| \leq 2} \sup _{y \in \mathbb{T}^{3}} \sup _{\xi \in \mathbb{Z}^{3}}\left|\partial_{y}^{\alpha} a(y, \xi)\right|^{2} .
$$

\section{Bounds For the Green's Functions and Trunchtion}

We collect some auxiliary results proved in [18]:

We have the following lower bound for the $L^{2}$-norm of the Green's function:

$$
\left\|G_{\lambda}\right\|_{2}^{2} \gg \lambda^{1 / 2-\varepsilon}
$$

For $L>0$, define the truncated Green's function by

$$
G_{\lambda, L}(x)=-\frac{1}{8 \pi^{3}} \sum_{\left.|| \xi\right|^{2}-\lambda \mid<L} \frac{e^{i \xi \cdot\left(x-x_{0}\right)}}{|\xi|^{2}-\lambda}
$$

and the $L^{2}$-normalized truncated Green's function by $g_{\lambda, L}=\frac{G_{\lambda, L}}{\left\|G_{\lambda, L}\right\|_{2}}$.

For $L=\lambda^{\delta}, \delta>0$, we have

$$
\left\|g_{\lambda}-g_{\lambda, L}\right\|_{2} \rightarrow 0
$$


as $\lambda \rightarrow \infty$ and

$$
\left\|G_{\lambda, L}\right\|_{2}=\left\|G_{\lambda}\right\|_{2}(1+o(1)) .
$$

It is also proved that for all $f \in C^{\infty}\left(\mathbb{T}^{3}\right)$

$$
\left|\left\langle f g_{\lambda}, g_{\lambda}\right\rangle-\left\langle f g_{\lambda, L}, g_{\lambda, L}\right\rangle\right| \leq 2\|f\|_{\infty}\left\|g_{\lambda}-g_{\lambda, L}\right\|_{2}
$$

and a similar proof shows that for all $a \in C^{\infty}\left(S^{*} \mathbb{T}^{3}\right)$

$$
\begin{gathered}
\left|\left\langle\mathrm{Op}(a) g_{\lambda}, g_{\lambda}\right\rangle-\left\langle\mathrm{Op}(a) g_{\lambda, L}, g_{\lambda, L}\right\rangle\right| \leq \\
2\|\mathrm{Op}(a)\|_{L^{2}\left(\mathbb{T}^{3}\right) \rightarrow L^{2}\left(\mathbb{T}^{3}\right)}\left\|g_{\lambda}-g_{\lambda, L}\right\|_{2} .
\end{gathered}
$$

\section{Basis Elements}

The following lemma shows that we can approximate a smooth function on $\mathbb{T}^{3} \times S^{2}$ by a finite linear combination of functions of the form $e_{\zeta, l, m}(x, \xi)=$ $Y_{l, m}(\xi) e^{i x \cdot \zeta}$, where $Y_{l, m}(\xi)$ is the spherical harmonic of degree $l$ and order $m$ (normalized so that $\int_{S^{2}}\left|Y_{l, m}\right|^{2} \mathrm{~d} \sigma=1$ ).

Lemma 5.1. For all $a \in C^{\infty}\left(\mathbb{T}^{3} \times S^{2}\right), \varepsilon>0$, there exist $N_{1}, N_{2}$ and

$$
P(x, \xi)=\sum_{|\zeta| \leq N_{1}} \sum_{l \leq N_{2}} \sum_{|m| \leq l} c_{\zeta, l, m} e_{\zeta, l, m}(x, \xi) \in C^{\infty}\left(\mathbb{T}^{3} \times S^{2}\right),
$$

such that for all $x \in \mathbb{T}^{3}, \xi \in S^{2}$ and for all multi-index $\alpha$ such that $|\alpha| \leq 2$, we have $\left|\partial_{x}^{\alpha}(a-P)(x, \xi)\right|<\varepsilon$.

Proof. Let $\varepsilon>0$. Expanding $a$ to its Fourier series in the variable $x$, for every $\xi \in S^{2}$ we have

$$
a(x, \xi)=\sum_{\zeta \in \mathbb{Z}^{3}} a_{\zeta}(\xi) e^{i x \cdot \zeta}
$$

where $a_{\zeta}(\xi)=\frac{1}{8 \pi^{3}} \int_{\mathbb{T}^{3}} a(x, \xi) e^{-i x \cdot \zeta} \mathrm{d} x$, with convergence in the sense of $L^{2}\left(\mathbb{T}^{3}\right)$. Moreover, since $a \in C^{\infty}\left(\mathbb{T}^{3} \times S^{2}\right)$, integration by parts yields that $a_{\zeta}(\xi) \ll|\zeta|^{-k}$ for all $k$ (where the implied constant is independent of $\xi$ ), so the series in (5.1) is uniformly convergent in $\mathbb{T}^{3} \times S^{2}$. Since uniform convergence implies $L^{2}$ convergence to the same (equivalence class of) function, and since for every $\xi$ both sides of (5.1) are continuous functions on $\mathbb{T}^{3}$, we conclude that the series uniformly converges in $\mathbb{T}^{3} \times S^{2}$ to $a(x, \xi)$. We also get that for every multi-index $\alpha$, we have

$$
\begin{aligned}
\partial_{x}^{\alpha} a(x, \xi) & =\sum_{\zeta \in \mathbb{Z}^{3}} a_{\zeta}(\xi) \partial_{x}^{\alpha} e^{i x \cdot \zeta} \\
& =\sum_{\zeta \in \mathbb{Z}^{3}} a_{\zeta}(\xi)(i \zeta)^{\alpha} e^{i x \cdot \zeta}
\end{aligned}
$$

and the series in (5.2) is uniformly convergent in $\mathbb{T}^{3} \times S^{2}$ to $\partial_{x}^{\alpha} a(x, \xi)$, so we can find $N_{1}$ such that for all $x, \xi$ and for all multi-index $\alpha$ such that $|\alpha| \leq 2$ 
we have

$$
\left|\partial_{x}^{\alpha}\left(a(x, \xi)-\sum_{|\zeta| \leq N_{1}} a_{\zeta}(\xi) e^{i x \cdot \zeta}\right)\right|<\frac{\varepsilon}{2} .
$$

For every $\zeta$, we have the spherical harmonics expansion:

$$
a_{\zeta}(\xi)=\sum_{l=0}^{\infty} \sum_{|m| \leq l} c_{\zeta, l, m} Y_{l, m}(\xi)
$$

where $c_{\zeta, l, m}=\int_{S^{2}} a_{\zeta}(\xi) \overline{Y_{l, m}(\xi)} \mathrm{d} \sigma$, with convergence in the sense of $L^{2}\left(S^{2}\right)$. Since $a(x, \xi) \in C^{\infty}\left(\mathbb{T}^{3} \times S^{2}\right)$, we easily see that for all $\zeta$ we have $a_{\zeta}(\xi) \in$ $C^{\infty}\left(S^{2}\right)$, and hence $\sum_{|m| \leq l} c_{\zeta, l, m} Y_{l, m}(\xi) \ll l^{-k}$ for all $k$ (see [2], for example), so the sum in (5.3) is uniformly convergent in $S^{2}$ for all $\zeta$, and again, it must converge to $a_{\zeta}(\xi)$. We conclude that there exists $N_{2}$ such that for all $|\zeta| \leq N_{1}$ and for all $\xi$ we have

$$
\left|a_{\zeta}(\xi)-\sum_{l \leq N_{2}} \sum_{|m| \leq l} c_{\zeta, l, m} Y_{l, m}(\xi)\right|<\frac{\varepsilon}{2 N_{1}^{5}}
$$

and if we denote

$$
P(x, \xi)=\sum_{|\zeta| \leq N_{1}} \sum_{l \leq N_{2}} \sum_{|m| \leq l} c_{\zeta, l, m} e_{\zeta, l, m}(x, \xi)
$$

we get that for all $x, \xi$ and for all multi-index $\alpha$ such that $|\alpha| \leq 2$ we have

$$
\begin{gathered}
\left|\partial_{x}^{\alpha}(a-P)(x, \xi)\right| \leq\left|\partial_{x}^{\alpha}\left(P(x, \xi)-\sum_{|\zeta| \leq N_{1}} a_{\zeta}(\xi) e^{i x \cdot \zeta}\right)\right|+\frac{\varepsilon}{2} \\
=\left|\partial_{x}^{\alpha}\left(\sum_{|\zeta| \leq N_{1}}\left(a_{\zeta}(\xi)-\sum_{l \leq N_{2}} \sum_{|m| \leq l} c_{\zeta, l, m} Y_{l, m}(\xi)\right) e^{i x \cdot \zeta}\right)\right|+\frac{\varepsilon}{2} \\
\leq N_{1}^{2} \sum_{|\zeta| \leq N_{1}}\left|a_{\zeta}(\xi)-\sum_{l \leq N_{2}} \sum_{|m| \leq l} c_{\zeta, l, m} Y_{l, m}(\xi)\right|+\frac{\varepsilon}{2}<\varepsilon .
\end{gathered}
$$

We can think of $e_{\zeta, l, m}(x, \xi)=Y_{l, m}(\xi) e^{i x \cdot \zeta}$ as a zero-th order positively homogeneous symbol in $S_{1,0}^{0}\left(\mathbb{T}^{3} \times \mathbb{R}^{3}\right)$, by extending $Y_{l, m}(\xi),(l, m) \neq(0,0)$ homogeneously to the domain $|\xi| \geq 1$, and arbitrarily to the domain $|\xi|<1$; the function $Y_{0,0}(\xi)=\frac{1}{2} \sqrt{\frac{1}{\pi}}$ extends to $\xi \in \mathbb{R}^{3}$ in an obvious way . In order to prove our main theorem, we will now see that it suffices to show a simpler version of it on the functions $e_{\zeta, l, m}(x, \xi)$. 
Proposition 5.2. Suppose that there is a subset $\Lambda_{\infty} \subseteq \Lambda$ of density one so that for all $\zeta, l, m$ with at least one of them nonzero we have

$$
\left\langle O p\left(e_{\zeta, l, m}\right) g_{\lambda, L}, g_{\lambda, L}\right\rangle \rightarrow 0
$$

as $\lambda \rightarrow \infty$ along $\Lambda_{\infty}$, then theorem 1.2 follows. Here $0<\delta<1, L=\lambda^{\delta}$.

Proof. Let $\varepsilon>0$, and $a(x, \xi) \in C^{\infty}\left(S^{*} \mathbb{T}^{3}\right)$. From lemma 5.1, there is

$$
P(x, \xi)=\sum_{|\zeta| \leq N_{1}} \sum_{l \leq N_{2}} \sum_{|m| \leq l} c_{\zeta, l, m} e_{\zeta, l, m}(x, \xi)
$$

such that for all $x \in \mathbb{T}^{3}, \xi \in \mathbb{R}^{3},|\xi| \geq 1$ and for all multi-index $\alpha$ such that $|\alpha| \leq 2$ we have $\left|\partial_{x}^{\alpha}(a-P)(x, \xi)\right|<\varepsilon$. Without loss of generality we can assume that we have

$$
\partial_{x}^{\alpha} P(x, 0)=\partial_{x}^{\alpha} a(x, 0)
$$

for all $x \in \mathbb{T}^{3}$ and for all multi-index $\alpha$, because for $\lambda$ large enough

$$
\mathrm{Op}(P) g_{\lambda, L}(x)=-\frac{1}{8 \pi^{3}\left\|G_{\lambda, L}\right\|_{2}} \sum_{|\xi|^{2}-\lambda \mid<L} e^{i\left(x-x_{0}\right) \cdot \xi} P(x, \xi) \frac{1}{|\xi|^{2}-\lambda}
$$

does not depend on the value of $P(x, 0)$ (and it is easy to change $P(x, \xi)$ in the domain $|\xi|<1$ to get a new symbol $\tilde{P} \in C^{\infty}\left(S^{*} \mathbb{T}^{3}\right)$ satisfying (15.4) $)$, so under this assumption the inequality $\left|\partial_{x}^{\alpha}(a-P)(x, \xi)\right|<\varepsilon$ holds for every $\xi \in \mathbb{Z}^{n}$. Since

$$
\mathrm{Op}\left(e_{0,0,0}\right)=\frac{1}{2} \sqrt{\frac{1}{\pi}} \mathrm{id},
$$

and since for $(\zeta, l, m) \neq(0,0,0)$

$$
\left\langle\mathrm{Op}\left(e_{\zeta, l, m}\right) g_{\lambda, L}, g_{\lambda, L}\right\rangle \rightarrow 0
$$

as $\lambda \rightarrow \infty$ along $\Lambda_{\infty}$, we have

$$
\begin{aligned}
\left\langle\mathrm{Op}(P) g_{\lambda, L}, g_{\lambda, L}\right\rangle & =\sum_{|\zeta| \leq N_{1}} \sum_{l \leq N_{2}} \sum_{|m| \leq l} c_{\zeta, l, m}\left\langle\mathrm{Op}\left(e_{\zeta, l, m}\right) g_{\lambda, L}, g_{\lambda, L}\right\rangle \\
& \rightarrow \frac{1}{2} \sqrt{\frac{1}{\pi}} c_{0,0,0}
\end{aligned}
$$

as $\lambda \rightarrow \infty$ along $\Lambda_{\infty}$, and

$$
\begin{aligned}
\int_{S^{*} \mathbb{T}^{3}} P \mathrm{~d} \mu & =\frac{1}{\operatorname{area}\left(\mathbb{T}^{3}\right) \operatorname{area}\left(S^{2}\right)} \\
& \sum_{|\zeta| \leq N_{1}} \sum_{l \leq N_{2}} \sum_{|m| \leq l} c_{\zeta, l, m}\left(\int_{\mathbb{T}^{3}} e^{i x \cdot \zeta} \mathrm{d} m\right)\left(\int_{S^{2}} Y_{l, m}(\xi) \mathrm{d} \sigma\right) \\
& =\frac{1}{\operatorname{area}\left(\mathbb{T}^{3}\right) \operatorname{area}\left(S^{2}\right)} c_{0,0,0}\left(\int_{\mathbb{T}^{3}} 1 \mathrm{~d} m\right)\left(\int_{S^{2}} Y_{0,0}(\xi) \mathrm{d} \sigma\right) \\
& =\frac{1}{2} \sqrt{\frac{1}{\pi}} c_{0,0,0}
\end{aligned}
$$


SO

$$
\left\langle\mathrm{Op}(P) g_{\lambda, L}, g_{\lambda, L}\right\rangle \rightarrow \int_{S^{*} \mathbb{T}^{3}} P \mathrm{~d} \mu
$$

as $\lambda \rightarrow \infty$ along $\Lambda_{\infty}$. Thus for $\lambda \in \Lambda_{\infty}$ large enough we have

$$
\begin{aligned}
\left|\left\langle\mathrm{Op}(P) g_{\lambda}, g_{\lambda}\right\rangle-\int_{S^{*} \mathbb{T}^{3}} P \mathrm{~d} \mu\right| & <\varepsilon+\left|\left\langle\mathrm{Op}(P) g_{\lambda}, g_{\lambda}\right\rangle-\left\langle\mathrm{Op}(P) g_{\lambda, L}, g_{\lambda, L}\right\rangle\right| \\
& \leq \varepsilon+2\|\mathrm{Op}(P)\|_{L^{2}\left(\mathbb{T}^{3}\right) \rightarrow L^{2}\left(\mathbb{T}^{3}\right)}\left\|g_{\lambda}-g_{\lambda, L}\right\|_{2} \\
& <C \varepsilon .
\end{aligned}
$$

Using the bound in (3.1), we get that

$$
\begin{aligned}
\left|\left\langle\mathrm{Op}(a-P) g_{\lambda}, g_{\lambda}\right\rangle\right|^{2} & \leq\left\|\mathrm{Op}(a-P) g_{\lambda}\right\|_{2}^{2} \\
& \leq\|\mathrm{Op}(a-P)\|_{L^{2}\left(\mathbb{T}^{3}\right) \rightarrow L^{2}\left(\mathbb{T}^{3}\right)}^{2} \\
& \leq C \sum_{|\alpha| \leq 2} \sup _{y \in \mathbb{T}^{3}} \sup _{\xi \in \mathbb{Z}^{3}}\left|\partial_{y}^{\alpha}(a-P)(y, \xi)\right|^{2} \\
& <C \varepsilon^{2}
\end{aligned}
$$

for $\lambda \in \Lambda_{\infty}$ (we call all our constants $C$ ).

We conclude that for $\lambda \in \Lambda_{\infty}$ large enough we have

$$
\begin{aligned}
\left|\left\langle\mathrm{Op}(a) g_{\lambda}, g_{\lambda}\right\rangle-\int_{S^{* \mathbb{T}^{3}}} a \mathrm{~d} \mu\right| & \leq\left|\left\langle\mathrm{Op}(a-P) g_{\lambda}, g_{\lambda}\right\rangle\right| \\
& +\left|\left\langle\mathrm{Op}(P) g_{\lambda}, g_{\lambda}\right\rangle-\int_{S^{*} \mathbb{T}^{3}} P \mathrm{~d} \mu\right| \\
& +\left|\int_{S^{*} \mathbb{T}^{3}} P \mathrm{~d} \mu-\int_{S^{*} \mathbb{T}^{3}} a \mathrm{~d} \mu\right| \\
& <C \varepsilon
\end{aligned}
$$

and the proposition follows.

\section{A Density One Set}

By the theorem of Legendre and Gauss (see [10]), the Diophantine equation

$$
x_{1}^{2}+x_{2}^{2}+x_{3}^{2}=n
$$

has solutions in integers $x_{i}(i=1,2,3)$ if and only if $n$ is not of the form $4^{a}(8 k+7)$ with $a \in \mathbb{Z}, a \geq 0$ and $k \in \mathbb{Z}$, and for all $n, r_{3}\left(4^{a} n\right)=r_{3}(n)$.

Equivalently, if we write $n=4^{a} n_{1}$, with $4 \nmid n_{1}$, then $n$ is a sum of three squares if and only if $n_{1} \not \equiv 7$ (8), that is to say

$$
\mathcal{N}_{3}=\left\{n \in \mathbb{N}: n=4^{a} n_{1}, 4 \nmid n_{1} \Rightarrow n_{1} \not \equiv 7(8)\right\},
$$

and $r_{3}(n)=r_{3}\left(n_{1}\right)$.

For $n \in \mathcal{N}_{3}$, write $n=4^{a} n_{1}$ with $4 \nmid n_{1}$, and define

$$
\mathcal{N}_{\text {good }}=\left\{n \in \mathcal{N}_{3}: n_{1}>n^{1 / 2}\right\}
$$


the set of "good" elements in $\mathcal{N}_{3}$, and $\mathcal{N}_{\text {bad }}=\mathcal{N}_{3} \backslash \mathcal{N}_{\text {good }}$ the set of "bad elements". We show that there are very few "bad" elements in $\mathcal{N}_{3}$ :

Lemma 6.1. \# $\left\{n \in \mathcal{N}_{\text {bad }}: n \leq X\right\} \leq X^{1 / 2} \log X$.

Proof. For $n \in \mathcal{N}_{b a d}, n \leq X$, we have $n=4^{a} n_{1}$ with $n_{1} \leq n^{1 / 2} \leq X^{1 / 2}$ and $a=\log _{4}\left(n / n_{1}\right) \leq \log n / \log 4 \leq \log X$, so there are at most $X^{1 / 2} \log X$ possibilities for such $n$.

For $\lambda \in \Lambda$, denote $n_{\lambda}$ to be the closest element in $\mathcal{N}_{3}$ to $\lambda$ (and if there are two elements with the same distance take the smallest of them). Note that $\left|n_{\lambda}-\lambda\right| \leq 1.5$, and for $n \neq n_{\lambda}$ we have $|n-\lambda| \geq 0.5$.

Define $\Lambda_{\infty}=\left\{\lambda \in \Lambda: n_{\lambda} \in \mathcal{N}_{\text {good }}\right\}$. We show that:

Lemma 6.2. $\Lambda_{\infty}$ is a density one set in $\Lambda$.

Proof. For every $\lambda \in \Lambda \backslash \Lambda_{\infty}$, we have $n_{k}<\lambda<n_{k+1}$, where either $n_{\lambda}=n_{k}$ or $n_{\lambda}=n_{k+1}$, and $n_{\lambda} \in \mathcal{N}_{\text {bad }}$. Thus, for every $n \in \mathcal{N}_{\text {bad }}$ such that $n \leq X+1.5$ there are at most two $\lambda \in \Lambda \backslash \Lambda_{\infty}, \lambda \leq X$ such that $n_{\lambda}=n$, so by lemma 6.1 we have

$$
\#\left\{\lambda \in \Lambda \backslash \Lambda_{\infty}: \lambda \leq X\right\} \leq 2 \#\left\{n \in \mathcal{N}_{\text {bad }}: n \leq X+1.5\right\} \ll X^{1 / 2} \log X
$$

but

$$
\#\{\lambda \in \Lambda: \lambda \leq X\} \geq \#\{n \leq X: n \not \equiv 0,4,7(8)\} \asymp X
$$

so

$$
\frac{\#\left\{\lambda \in \Lambda \backslash \Lambda_{\infty}: \lambda \leq X\right\}}{\#\{\lambda \in \Lambda: \lambda \leq X\}} \ll X^{-1 / 2} \log X
$$

which tends to zero as $X \rightarrow \infty$, so $\Lambda \backslash \Lambda_{\infty}$ is a density zero set in $\Lambda$, and therefore $\Lambda_{\infty}$ is a density one set in $\Lambda$.

\section{Proving Theorem 1.2}

We are only left to prove the following proposition:

Proposition 7.1. Let $\zeta, l, m$ with at least one of them nonzero, and let $0<\delta<1 / 28, L=\lambda^{\delta}$. Then

$$
\left\langle O p\left(e_{\zeta, l, m}\right) g_{\lambda, L}, g_{\lambda, L}\right\rangle \rightarrow 0
$$

as $\lambda \rightarrow \infty$ along $\Lambda_{\infty}$. 
Proof. We have

$$
\begin{gathered}
\asymp\left|\left\langle\sum_{\left.|| \xi\right|^{2}-\lambda \mid<L} \frac{e^{i\left(x-x_{0}\right) \cdot \xi}}{|\xi|^{2}-\lambda} e^{i x \cdot \zeta} Y_{l, m}\left(\frac{\xi}{|\xi|}\right), \sum_{\left.|| \eta\right|^{2}-\lambda \mid<L} \frac{e^{i\left(x-x_{0}\right) \cdot \eta}}{|\eta|^{2}-\lambda}\right\rangle\right| \\
\asymp\left|\sum_{\substack{\left.|| \xi\right|^{2}-\lambda|<L\\
||\xi+\zeta|^{2}-\lambda \mid<L}} \frac{1}{\left(|\xi|^{2}-\lambda\right)\left(|\xi+\zeta|^{2}-\lambda\right)} Y_{l, m}\left(\frac{\xi}{|\xi|}\right)\right| .
\end{gathered}
$$

First, assume that $\zeta \neq 0$ :

The functions $Y_{l, m}$ are bounded on $S^{2}$, so

$$
\left|\left\langle\operatorname{Op}\left(e_{\zeta, l, m}\right) G_{\lambda, L}, G_{\lambda, L}\right\rangle\right| \ll \sum_{\substack{\left.|| \xi\right|^{2}-\lambda|<L\\||\xi+\zeta|^{2}-\lambda \mid<L}} \frac{1}{\left.|| \xi\right|^{2}-\lambda|||\xi+\zeta|^{2}-\lambda \mid}
$$

and therefore

$$
\left|\left\langle\operatorname{Op}\left(e_{\zeta, l, m}\right) g_{\lambda, L}, g_{\lambda, L}\right\rangle\right| \ll \frac{\sum_{\left.|| \xi\right|^{2}-\lambda \mid<L}^{|| \xi+\left.\zeta\right|^{2}-\lambda \mid<L}}{\left\|G_{\lambda, L}\right\|_{2}^{2}} \rightarrow 0
$$

as $\lambda \rightarrow \infty$ by the proof of Proposition 3.9 in 18 .

Assume now that $\zeta=0$.

We have

$$
\begin{aligned}
\left|\left\langle\mathrm{Op}\left(e_{0, l, m}\right) G_{\lambda, L}, G_{\lambda, L}\right\rangle\right| & \asymp\left|\sum_{\left.|| \xi\right|^{2}-\lambda \mid<L} \frac{Y_{l, m}\left(\frac{\xi}{|\xi|}\right)}{\left(|\xi|^{2}-\lambda\right)^{2}}\right| \\
& =\left|\sum_{|n-\lambda|<L} \frac{W_{l, m}(n)}{(n-\lambda)^{2}}\right|
\end{aligned}
$$

where

$$
W_{l, m}(n)=\sum_{|\xi|^{2}=n} Y_{l, m}\left(\frac{\xi}{|\xi|}\right) .
$$

If we write $n=4^{a} n_{1}$ with $4 \nmid n_{1}$, then by Duke's estimate (see [5, 6])

$$
\left|W_{l, m}(n)\right|=\left|W_{l, m}\left(n_{1}\right)\right| \ll n_{1}^{13 / 28+\varepsilon} \leq n^{13 / 28+\varepsilon}
$$

and by Siegel's theorem [17] $r_{3}(n)=r_{3}\left(n_{1}\right) \gg n_{1}^{1 / 2-\varepsilon}$, so

$$
\frac{\left|W_{l, m}(n)\right|}{r_{3}(n)} \ll n_{1}^{-1 / 28+\varepsilon} \text {. }
$$


For $\lambda \in \Lambda_{\infty}$, recall that $n_{\lambda}$ is the closest element in $\mathcal{N}_{3}$ to $\lambda$ (and if there are two elements with the same distance take the smallest of them). From the definition of $\Lambda_{\infty}$ we know that $n_{\lambda} \in \mathcal{N}_{\text {good }}$.

Write

$$
\sum_{|n-\lambda|<L} \frac{W_{l, m}(n)}{(n-\lambda)^{2}}=\sum_{\substack{|n-\lambda|<L \\ n \neq n_{\lambda}}} \frac{W_{l, m}(n)}{(n-\lambda)^{2}}+\frac{W_{l, m}\left(n_{\lambda}\right)}{\left(n_{\lambda}-\lambda\right)^{2}} .
$$

Since for $n \neq n_{\lambda}$ we have $|n-\lambda| \geq 0.5$ :

$$
\left|\sum_{\substack{|n-\lambda|<L \\ n \neq n_{\lambda}}} \frac{W_{l, m}(n)}{(n-\lambda)^{2}}\right| \ll \sum_{|n-\lambda|<L} n^{13 / 28+\varepsilon} \ll \lambda^{13 / 28+\delta+\varepsilon}
$$

so

$$
\frac{\left|\sum_{\substack{|n-\lambda|<L \\ n \neq n_{\lambda}}} \frac{W_{l, m}(n)}{(n-\lambda)^{2}}\right|}{\left\|G_{\lambda, L}\right\|_{2}^{2}} \ll \frac{\lambda^{13 / 28+\delta+\varepsilon}}{\left\|G_{\lambda}\right\|_{2}^{2}} \ll \lambda^{-1 / 28+\delta+2 \varepsilon}
$$

which tends to zero for $\varepsilon>0$ small enough, since $\delta<\frac{1}{28}$. For the other term, writing $n_{\lambda}=4^{a} n_{1}$ with $4 \nmid n_{1}$, we have

$$
\begin{aligned}
\frac{\left|\frac{W_{l, m}\left(n_{\lambda}\right)}{\left(n_{\lambda}-\lambda\right)^{2}}\right|}{\left\|G_{\lambda, L}\right\|_{2}^{2}} & \asymp \frac{\frac{\left|W_{l, m}\left(n_{\lambda}\right)\right|}{\left(n_{\lambda}-\lambda\right)^{2}}}{\left\|G_{\lambda}\right\|_{2}^{2}} \\
& \asymp \frac{\frac{\left|W_{l, m}\left(n_{\lambda}\right)\right|}{\left(n_{\lambda}-\lambda\right)^{2}}}{\sum_{n=0}^{\infty} \frac{r_{3}(n)}{(n-\lambda)^{2}}} \\
& \ll \frac{\left|W_{l, m}\left(n_{\lambda}\right)\right|}{r_{3}\left(n_{\lambda}\right)} \ll n_{1}^{-1 / 28+\varepsilon}
\end{aligned}
$$

and since $n_{\lambda} \in \mathcal{N}_{\text {good }}$, we have

$$
n_{1}^{-1 / 28+\varepsilon} \leq n_{\lambda}^{-1 / 56+\frac{\varepsilon}{2}} \ll \lambda^{-1 / 56+\frac{\varepsilon}{2}}
$$

so this term also tends to zero as $\lambda \rightarrow \infty$ along $\Lambda_{\infty}$.

\section{REFERENCES}

[1] Agranovich, M.S.: Spectral properties of elliptic pseudodifferential operators on a closed curve. (Russian) Funktsional. Anal. i Prilozhen. 13, no. 4, 54-56 (1979) (English translation in Functional Analysis and Its Applications. 13, p. 279-281 (1979))

[2] Atkinson, K., Han, W.: Spherical Harmonics and Approximations on the Unit Sphere: An Introduction. Springer (2012)

[3] Colin de Verdière, Y.: Pseudo-laplaciens I. Annales de l'Institut Fourier, tome 32, no. 3, 275-286 (1982) 
[4] Colin de Verdière, Y.: Ergodicité et fonctions propres du laplacien. Comm. Math. Phys. 102, 497-502 (1985)

[5] Duke, W.: Hyperbolic distribution problems and half-integral weight Maass forms. Invent. Math. 92, 73-90 (1988)

[6] Duke, W., Schulze-Pillot, R.: Representation of integers by positive ternary quadratic forms and equidistribution of lattice points on ellipsoids. Invent. Math. 99, 49-57 (1990)

[7] Erdős, P., Hall, R.R.: On the angular distribution of Gaussian integers with fixed norm. Discrete Mathematics 200, 87-94 (1999)

[8] Golubeva, E.P., Fomenko, O.M.: Asymptotic distribution of integral points on the three-dimensional sphere. Zap. Nauchn. Sem. Leningr. Otd. Mat. Inst. Akad. Nauk SSSR, 160, 54-71 (1987) (English translation in Journal of Soviet Mathematics 52, Issue 3, 3036-3048 (1990))

[9] Golubeva, E.P., Fomenko, O.M.: Remark on asymptotic distribution of the integral points on the large three-dimensional sphere . Zap. Nauchn. Sem. Leningr. Otd. Mat. Inst. Akad. Nauk SSSR, 185, 22-28 (1990) (English translation in Journal of Soviet Mathematics 59, Issue 6, 1148-1152 (1992))

[10] Grosswald, E.: Representations of Integers as Sums of Squares. Springer-Verlag, NewYork (1985)

[11] McLean, W.: Local and Global Descriptions of Periodic Pseudodifferential Operators. Math. Nachr. 150, 151-161 (1991)

[12] Rudnick, Z., Ueberschär, H.: Wave function statistics for a point scatterer on the torus. Comm. Math. Phys. 316, 763-782 (2012)

[13] Ruzhansky, M., Turunen, V.: Pseudo-Differential Operators and Symmetries: Background Analysis and Advanced Topics. Birkhäuser (2010)

[14] Schnirelman, A.: Ergodic properties of eigenfunctions. Usp. Math. Nauk. 29, 181-182 (1974)

[15] Shigehara, T.: Conditions for the appearance of wave chaos in quantum singular systems with a pointlike scatterer. Phys. Rev. E 50, 4357-4370 (1994)

[16] Shigehara, T., Cheon, T.: Spectral properties of three-dimensional quantum billiards with a pointlike scatterer. Phys. Rev. E 55, 6832-6844 (1997)

[17] Siegel, C. L.: Über die Classenzahl quadratischer Zahlkörper. Acta Arith. 1 , 83-86 (1935)

[18] Yesha, N.: Eigenfunction Statistics for a Point-Scatterer on a Three-Dimensional Torus. Annales Henri Poincaré 14, Issue 7, 1801-1836 (2013)

[19] Zelditch, S.: Uniform distribution of eigenfunctions on compact hyperbolic surfaces. Duke Math. J. 55, 919-941 (1987)

Raymond and Beverly Sackler School of Mathematical Sciences, Tel Aviv University, Tel AVIV 69978, IsRAel

E-mail address: nadavye1@post.tau.ac.il 